\title{
Modelo de Referência e Método de Avaliação para Melhoria de Processo de Software - versão 1.0 (MR-MPS e MA-MPS)
}

\author{
Kival Chaves Weber ${ }^{1}$, Eratóstenes Araújo ${ }^{1}$, Cristina Angela Filipak Machado², \\ Danilo Scalet ${ }^{2}$, Clênio Figueiredo Salviano ${ }^{3}$, Ana Regina Cavalcanti da Rocha ${ }^{4}$ \\ ${ }^{1}$ SOFTEX - Associação para Promoção da Excelência do Software Brasileiro \\ ${ }^{2}$ CELEPAR - Companhia de Informática do Paraná \\ ${ }^{3}$ CenPRA - Centro de Pesquisas Renato Archer \\ ${ }^{4}$ COPPE/UFRJ - Universidade Federal do Rio de Janeiro \\ \{kival.weber, era\}@nac.softex.br, \{cristina, danilo\}@pr.gov.br, \\ clenio.salviano@cenpra.gov.br, darocha@cos.ufrj.br
}

\begin{abstract}
This paper describes a Process Reference Model (MR-MPS) and a Process Assessment Model (MA-MPS), that are being developed in the MPS.BR Project - a Brazilian software process improvement project. MRMPS and MA-MPS were created according to the Brazilian reality and they aim at the full compatibility with CMMI and the conformance with ISO/IEC 12207 and ISO/IEC 15504. The MPS Model is described in three documents: a general guide, an assessment guide, and an acquisition guide. This paper also presents the initial outcomes of the project. The MPS Model has a great potential to be replicated to many cities in Brazil and other countries with similar software industry characteristics, such as in Latin America.
\end{abstract}

Resumo. Este artigo descreve um Modelo de Referência de Processo e um Modelo de Avaliação de Processo, denominados MR-MPS e MA-MPS, que estão sendo desenvolvidos no Projeto MPS.BR - melhoria de processo do sofware Brasileiro. O MR-MPS e o MA-MPS foram criados de acordo com a realidade brasileira e visam tanto a plena compatibilidade com o CMMI como a conformidade com as normas ISO/IEC 12207 e ISO/IEC 15504. O Modelo MPS é descrito em três documentos: guia geral, guia de avaliação e guia de aquisição. Este artigo também apresenta os primeiros resultados do projeto. O Modelo MPS tem um grande potencial de replicabilidade em muitas cidades no Brasil e em outros países com características similares no que se refere ao setor de software, como na América Latina.

\section{Introdução}

Estudos mostram que é necessário um esforço significativo para aumentar a maturidade dos processos de software nas empresas brasileiras e que, nos últimos anos, as empresas de software no Brasil favoreceram a ISO 9000, em detrimento de outras normas e modelos especificamente voltadas para a melhoria de processos de software [MCT/SEPIN 2001 e Veloso 2003]. 


\section{Simpósio Brasileiro de Qualidade de Software}

Segundo dados do Ministério da Ciência e Tecnologia, em 2003, 214 empresas que desenvolviam software no Brasil tinham certificação ISO 9000, enquanto eram trinta as empresas com certificado SEI/CMU de avaliações CMM. Estas últimas, a maioria subsidiária no país de grandes empresas multinacionais, comprendiam em 2003: 24 empresas no nível 2, cinco no nível 3, uma no nível 4 e nenhuma no nível 5 do CMM.

Para ajudar na solução deste problema, em Dez 2003, a SOFTEX - Associação para Promoção da Excelência do Software Brasileiro propôs o Projeto MPS Br melhoria de processo do software Brasileiro [Weber et al 2004a e 2004b]. A SOFTEX é uma entidade privada, sem fins lucrativos, que promove ações com abrangência nacional visando transformar o Brasil em um centro de excelência mundial na produção e exportação de software. Dispõe de 31 Agentes SOFTEX, localizados em 23 cidades de 13 Unidades da Federação, com mais de mil e setenta empresas associadas - com o seguinte porte segundo o número de funcionários: 11\% grandes, $13 \%$ médias, 36\% pequenas e $40 \%$ micro empresas, conforme descrito em www.softex.br.

Em 2004, o Projeto MPS.BR foi desenvolvido com recursos próprios das seguintes instituições-âncora, integrantes do Comitê Gestor MPS: SOFTEX (coordenadora do projeto), COPPE/UFRJ (coordenadora da ETM - Equipe Técnica do Modelo) e RIOSOFT no Rio de Janeiro/RJ, CenPRA e Agente SOFTEX em Campinas/SP, CESAR em Recife/PE e CELEPAR em Curitiba/PR. Em 2005-2006, o projeto conta com apoio financeiro do MCT através de recursos do FNDCT/CT-INFO/ VERDE-AMARELO/FINEP.

O Projeto MPS.BR visa a melhoria de processo do software Brasileiro, a um custo acessível, e compreende duas metas:

- desenvolvimento e aprimoramento do Modelo MPS, compatível com o CMMI e em conformidade com as normas ISO/IEC 12207 e ISO/IEC 15504;

- a implementação e avaliação do Modelo MPS, a um custo acessível em todas as regiões do país, com foco em grupos de pequenas e médias empresas (PMEs).

Não é objetivo do Projeto MPS.BR definir algo novo no que se refere a normas e modelos. Sua novidade está na estratégia de implementação, criada para a realidade brasileira [Weber et al 2004a e 2004b].

Este artigo apresenta a versão 1.0 do Modelo de Referência e do Método de Avaliação para melhoria de processo de software (MR-MPS e MA-MPS). A seção 2 resume o Projeto MPS.BR e suas duas metas para o biênio 2005-2006. Na seção 3 são descritos o MR-MPS e o MA-MPS, detalhados em três guias: Guia Geral, Guia de Avaliação e Guia de Aquisição. Na seção 4 são apresentados os resultados iniciais do projeto, alcançados de dezembro de 2003 a março de 2005. Na seção 5, como conclusão, são apresentadas as considerações finais deste artigo.

\section{Projeto MPS.BR - melhoria de processo do software Brasileiro}

As organizações e associações industriais que percebem uma vantagem competitiva estratégica nos seus processos de negócio, agora, tem a opção de criar o seu próprio Modelo de Referência de Processo ou usar um modelo de processo existente. Daí, podem criar um Modelo de Avaliação de Processo baseado neste modelo de referência. 


\section{Simpósio Brasileiro de Qualidade de Software}

Entretanto, o esforço necessário para assegurar que os modelos estão de acordo ou em conformidade com a ISO/IEC 15504 não pode ser subestimado. É provável que apenas grandes organizações possam assumir um empreendimento desta magnitude. $\mathrm{Na}$ Europa, as indústrias espacial e automotiva estão seguindo este caminho através de suas associações industriais [van Loon 2004a].

Este foi o desafio assumido pela SOFTEX no Brasil, como associação nacional da indústria de software e serviços correlatos, ao criar o Projeto MPS.BR em Dez 2003. Em termos de organização e gestão, o projeto possui:

- um Comitê Gestor (CG), coordenado pela SOFTEX;

- uma Equipe Técnica do Modelo (ETM), coordenada pela COPPE/UFRJ;

- um Fórum de Credenciamento e Controle (FCC), para tratar de autorizações SOFTEX das Instituições Implementadoras e Instituições Avaliadoras do Modelo MPS (II-MPS e IA-MPS).

Como vimos, o objetivo do projeto é a melhoria de processo do software em todo o país, com foco nas PMEs, a um custo acessível. O Projeto MPS.BR tem duas metas, a primeira de desenvolvimento tecnológico e a segunda de melhoria de processo de software nas empresas:

$>$ Meta Física 1: Desenvolvimento e Aprimoramento do Modelo MPS

- Em 2005-2006: 3 Guias MPS, Cursos e Provas MPS anuais, 20 Instituições Implementadoras do Modelo MPS (II-MPS) e 15 Instituições Avaliadoras do Modelo MPS (IA-MPS).

Meta Física 2: Implementação e Avaliação do Modelo MPS em Empresas

- Em 2005-2006: 120 empresas com Modelo MPS implementado e 60 empresas avaliadas.

- Em 2007-2008: 180 empresas com Modelo MPS implementado e 80 empresas avaliadas, adicionalmente.

\section{Descrição do Modelo MPS}

O Modelo MPS é um modelo de melhoria e avaliação de processo de software, destinado preferencialmente a pequenas e médias empresas, normalmente grupos de empresas em um Modelo de Negócio Cooperado (MNC); mas, também, vem sendo aplicado em organizações de grande porte no Modelo de Negócio Específico (MNE). Desta forma, o modelo visa atender às necessidades de negócio das empresas e ser reconhecido, nacional e internacionalmente, como um modelo aplicável nas organizações que desenvolvem e adquirem software.

A base técnica utilizada para a construção do Modelo MPS foram as normas ISO/IEC 12207 e ISO/IEC 15504 - com as quais está em conformidade. Complementarmente, o modelo cobre o conteúdo de outros Modelos de Referência de Processo, como o CMMI [Chrissis et al 2003] - com o qual é compatível. O modelo também define regras para sua implementação e avaliação, de modo a dar sustentação e garantia de que está sendo empregado de forma coerente com as suas definições. Como mostra a Figura 1, o Modelo MPS possui três componentes: Modelo de Referência para 


\section{Simpósio Brasileiro de Qualidade de Software}

Melhoria de Processo de Software (MR-MPS), Método de Avaliação para Melhoria de Processo de Software (MA-MPS) e Modelo de Negócio para Melhoria de Processo de Software (MN-MPS). Cada componente do modelo foi descrito através de documentos específicos, tais como os três guias.

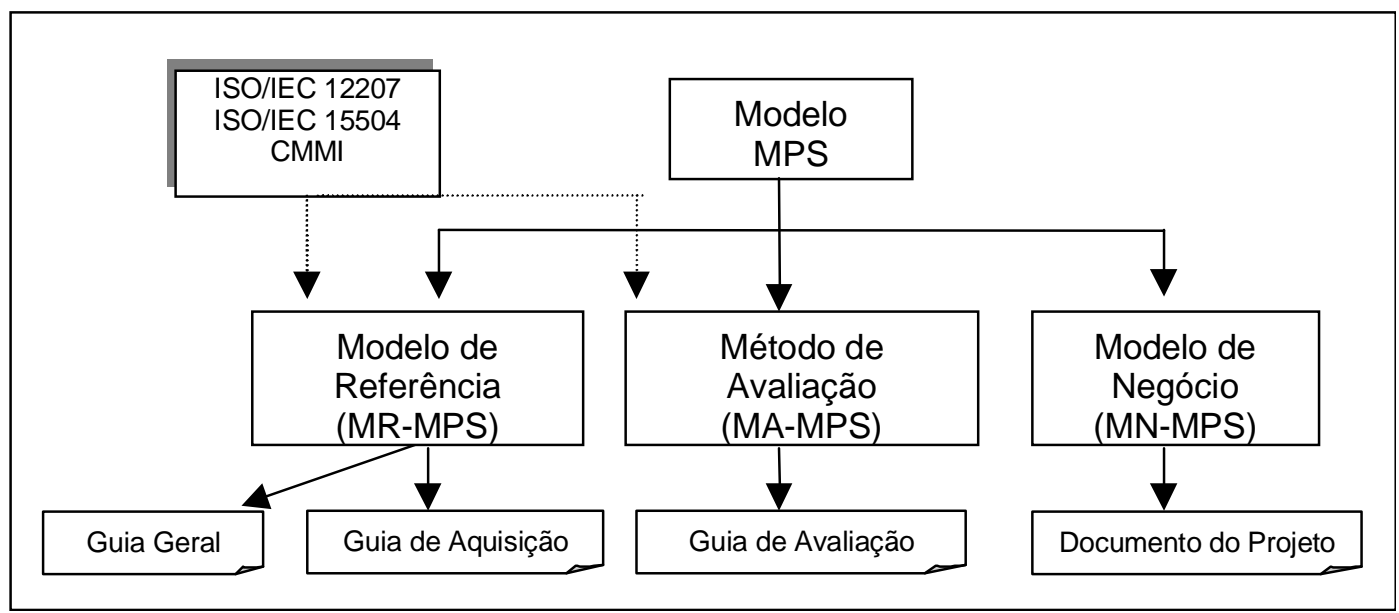

Figura 1. Modelo MPS

O Modelo de Referência para Melhoria de Processo de Software (MR-MPS) contém os requisitos que as organizações devem atender para estar em conformidade com o Modelo MPS. O MR-MPS contém as definições dos níveis de maturidade e capacidade de processos, e dos processos em si. O MR-MPS foi baseado nas normas ISO/IEC 12207 e ISO/IEC 15504 e adequado ao CMMI. O MR-MPS está descrito no Guia Geral.

O Guia de Aquisição é um documento complementar destinado a organizações que queiram adquirir software e serviços correlatos com base em normas internacionais e em práticas recomendadas internacionalmente. O Guia de Aquisição não contém requisitos do MR-MPS, mas boas práticas de aquisição de software e serviços correlatos.

O Método de Avaliação para Melhoria de Processo de Software (MA-MPS) descreve o processo de avaliação, os requisitos para os avaliadores e os requisitos para atender ao MR-MPS, em conformidade com a norma ISO/IEC 15504. O MA-MPS está descrito no Guia de Avaliação.

O Modelo de Negócio para Melhoria de Processo de Software (MN-MPS) contém uma descrição das regras de negócio para três domínios: o domínio do Projeto MPS.BR, coordenado pela SOFTEX; o domínio das Instituições Implementadoras do Modelo MPS e Instituições Avaliadoras do Modelo MPS, autorizadas pela SOFTEX (II-MPS e IA_MPS); e o domínio das empresas e organizações que queiram fazer uso do Modelo MPS para melhorar seus processos de software, seja em grupos de empresas no Modelo de Negócio Cooperado (MNC) seja uma empresa ou organização específica no Modelo de Negócio Específico (MNE). O detalhamento dessas regras de negócio está disponível na SOFTEX, como um documento do Projeto MPS.BR. 


\section{Simpósio Brasileiro de Qualidade de Software}

\subsection{Guia Geral MPS}

O documento Guia Geral contém uma descrição geral do Modelo MPS, definições comuns e o detalhamento do Modelo de Referência para Melhoria de Processo de Software (MR-MPS). O Guia Geral contém os requisitos que as organizações devem atender para estar em conformidade com o Modelo MPS.

O MR-MPS é definido através de sete níveis de maturidade, seqüenciais e acumulativos. Cada nível de maturidade é uma junção entre processos e capacidade dos processos, ou seja, é composto por um conjunto de processos em um determinado nível de capacidade.

Os processos e as capacidades dos processos foram descritos segundo as normas ISO/IEC 12207, e suas emendas 1 e 2, e ISO/IEC 15504-5. O progresso e o atendimento do nível de maturidade se obtém, quando são atendidos todos os resultados e propósito do processo; e os atributos de processo relacionados àquele nível. Esta estrutura é mostrada na Figura 2.

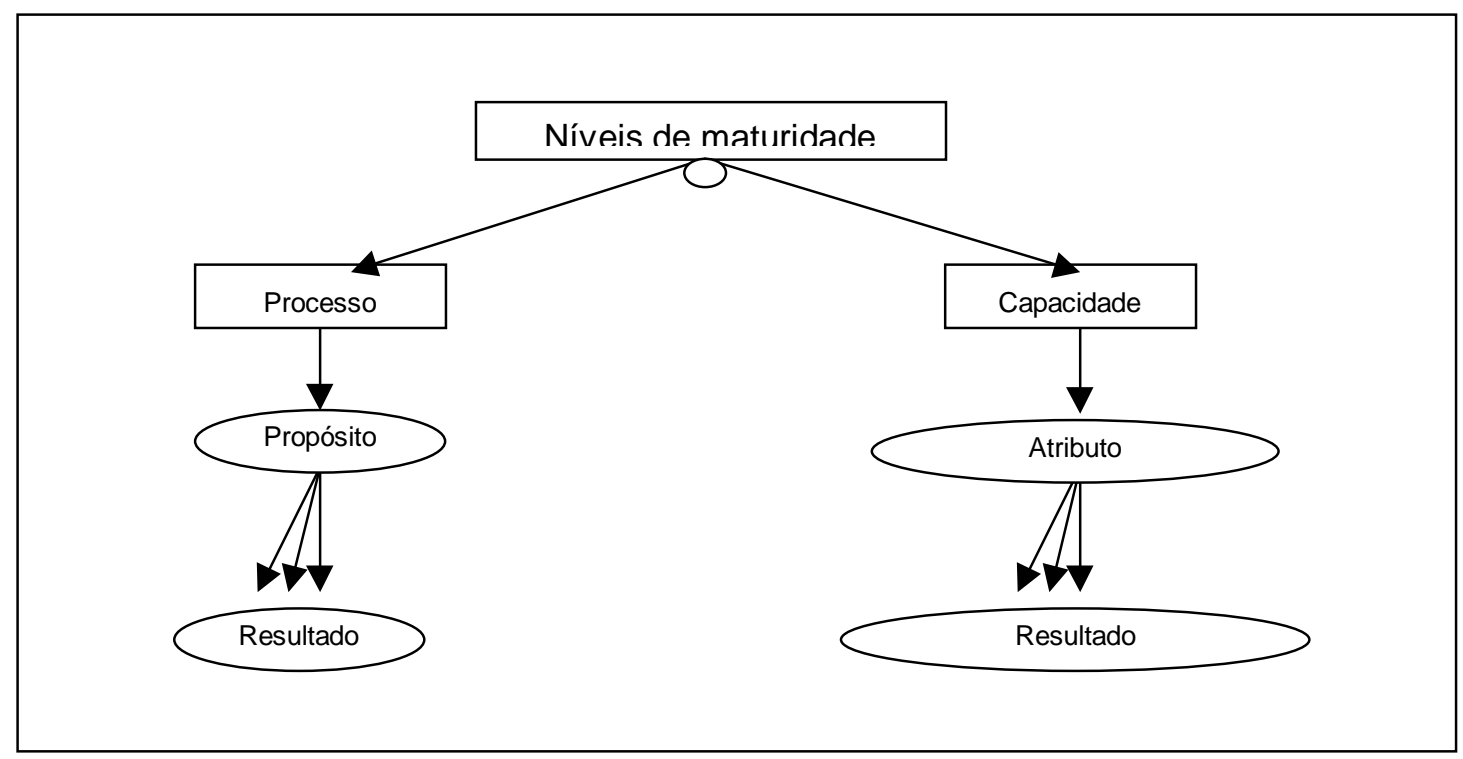

Figura 2. Estrutura do MR-MPS

Os níveis de maturidade estabelecem patamares de evolução de processos, caracterizando estágios de melhoria de implementação de processos na organização. O MR-MPS define sete níveis de maturidade: A (Em Otimização), B (Gerenciado Quantitativamente), C (Definido), D (Largamente Definido), E (Parcialmente Definido), F (Gerenciado) e G (Parcialmente Gerenciado). O nível G é o inicial, indicando que ele é mais imaturo que os demais níveis, e o nível A é o mais maduro. Os níveis de maturidade do MR-MPS são baseados nos quatro níveis de maturidade da representação por estágio do CMMI (níveis 2 a 5), sendo os níveis F, C, B e A do MRMPS correspondentes respectivamente aos níveis 2, 3, 4 e 5 do CMMI. O nível G é um nível intermediário entre os níveis 1 e 2 do CMMI e os níveis E e D são dois níveis intermediários entre os níveis 2 e 3 do CMMI.

Esta graduação em sete níveis do MR-MPS possibilita uma implementação e reconhecimento mais gradual da melhoria de processo de software, facilitando sua 
adequação às pequenas e médias empresas, com visibilidade dos resultados em prazos mais curtos. A correspondência entre os níveis do MR-MPS e os do CMMI permite que um mesmo esforço de melhoria seja reconhecido pelo MR-MPS e pelo CMMI.

A capacidade do processo possui cinco atributos de processos: AP $1.1-\mathrm{o}$ processo é executado; AP 2.1 - o processo é gerenciado; AP 2.2 - os produtos de trabalho do processo são gerenciados; AP 3.1 - o processo é definido; AP 3.2 - o processo-padrão está implementado. Estes atributos são baseados nos atributos de processo da ISO/IEC 15504 e correspondem às práticas genéricas do CMMI-SE/SW.

Os nomes e siglas dos processos e os atributos de processos, que devem ser acrescentados ao perfil de capacidade de processo para cada nível de maturidade do MR-MPS, são apresentados a seguir (ver Tabela 1).

Tabela 1. Níveis de Maturidade do MR-MPS

\begin{tabular}{|c|c|c|}
\hline Nível & Nome e Sigla dos Processos & $\begin{array}{c}\text { Atributos de } \\
\text { Processo } \\
\text { (Capacidade) }\end{array}$ \\
\hline $\begin{array}{c}\text { A } \\
\text { (mais } \\
\text { alto) } \\
\end{array}$ & $\begin{array}{l}\text { Inovação e Implantação na Organização - IIO, } \\
\text { Análise e Resolução de Causas - ARC }\end{array}$ & $\begin{array}{l}\text { AP } 1.1, \\
\text { AP } 2.1, \text { AP } 2.2, \\
\text { AP } 3.1 \text { e AP } 3.2 \\
\end{array}$ \\
\hline B & $\begin{array}{l}\text { Desempenho do Processo Organizacional - DEP, } \\
\text { Gerência Quantitativa do Projeto - GQP }\end{array}$ & \begin{tabular}{|l} 
AP 1.1, \\
AP 2.1, AP 2.2, \\
AP 3.1 e AP 3.2 \\
\end{tabular} \\
\hline $\mathrm{C}$ & $\begin{array}{l}\text { Gerência de Riscos - GRI, } \\
\text { Análise de Decisão e Resolução - ADR }\end{array}$ & $\begin{array}{l}\text { AP } 1.1, \\
\text { AP } 2.1, \text { AP } 2.2, \\
\text { AP } 3.1 \text { e AP } 3.2\end{array}$ \\
\hline $\mathrm{D}$ & $\begin{array}{l}\text { Desenvolvimento de Requisitos - DRE, } \\
\text { Solução Técnica - STE, } \\
\text { Validação - VAL, } \\
\text { Verificação - VER, } \\
\text { Integração do Produto - ITP, } \\
\text { Instalação do Produto - ISP, } \\
\text { Liberacão do Produto - LIP } \\
\end{array}$ & $\begin{array}{l}\text { AP 1.1, } \\
\text { AP 2.1, AP 2.2, } \\
\text { AP 3.1 e AP 3.2 }\end{array}$ \\
\hline $\mathrm{E}$ & $\begin{array}{l}\text { Treinamento - TRE, } \\
\text { Definição do Processo Organizacional - DFP, } \\
\text { Avaliação e Melhoria do Processo Organizacional - AMP, } \\
\text { Adaptação do Processo para Gerência de Projeto - APG } \\
\end{array}$ & $\begin{array}{l}\text { AP 1.1, } \\
\text { AP 2.1, AP 2.2, } \\
\text { AP 3.1 e AP 3.2 }\end{array}$ \\
\hline $\mathrm{F}$ & $\begin{array}{l}\text { Gerência de Configuração - GCO, } \\
\text { Garantia da Qualidade - GQA, } \\
\text { Medição - MED, } \\
\text { Aquisição - AQU }\end{array}$ & $\begin{array}{l}\text { AP } 1.1 \\
\text { AP } 2.1 \text { e AP } 2.2\end{array}$ \\
\hline $\begin{array}{c}\mathrm{G} \\
\text { (mais } \\
\text { baixo) }\end{array}$ & $\begin{array}{l}\text { Gerência de Projeto - GPR, } \\
\text { Gerência de Requisitos - GRE }\end{array}$ & AP 1.1 e AP 2.1 \\
\hline
\end{tabular}




\section{Simpósio Brasileiro de Qualidade de Software}

\subsection{Guia de Avaliação MPS}

\subsubsection{Avaliação de Processo}

Segundo Han van Loon [van Loon 2004b]:

- idealmente, a organização deve considerar o seu desempenho em relação aos concorrentes e pares no seu mercado e contra seus pares em mercados correlatos - em outras palavras, o "sucesso no seu negócio" como um todo. A organização poderia relacionar este desempenho aos processos (e à tecnologia) que usa, para determinar como estes processos contribuem para o seu sucesso nos negócios. Também, poderia considerar o seu desempenho absoluto (ou a falta de desempenho) e tentar calcular o custo desta falha;

- um processo de avaliação provê uma estrutura de medição, independente e aceita, para a avaliação de processos. A norma ISO/IEC 15504 vem sendo desenvolvida como uma visão internacional das "melhores práticas" para o processo de avaliação. O uso da ISO/IEC 15504 permite que as organizações avaliem se os seus processos são capazes de ajudá-las a alcançar o sucesso nos negócios.

\subsubsection{Método de Avaliação}

O documento Guia de Avaliação descreve o Método de Avaliação para Melhoria de Processo de Software (MA-MPS) e é composto basicamente pelos requisitos do método, atividades do método, indicadores para avaliação e características da qualificação dos avaliadores. Este método permite a realização de avaliações em unidades organizacionais segundo o Modelo MPS.

Os requisitos do MA-MPS são baseados nos requisitos do método de avaliação definidos na norma ISO/IEC 15504-2, os requisitos definidos no ARC do CMMI e os requisitos específicos do Projeto MPS.BR. Desta forma, o método é compatível com a ISO/IEC 15504 e ARC do CMMI, e adequado às pequenas e médias empresas.

As atividades do método de avaliação são baseadas principalmente no método Standard CMMI Appraisal Method for Process Improvement (SCAMPI) [SEI 2001], com elementos dos métodos QuickLocus [Kohan 2003] e MARES [Anacleto et al 2004].

O SCAMPI é o método de avaliação padrão para gerar classificação comparável para as representações por estágio e contínua do CMMI. O QuickLocus é um método de avaliação de processo voltado para organizações de software com até cinqüenta pessoas, utilizando um tempo reduzido de aplicação e cujo resultado fornece a base para um plano de melhoria do processo de desenvolvimento. O MARES é um método para execução da avaliação de processos de software em micro e pequenas empresas, em conformidade com a ISO/IEC 15504, com foco na melhoria dos processos.

O processo de avaliação é composto por quatro fases: Preparação e planejamento, Condução da avaliação, Resultados e Certificação. O fluxo destas fases, os principais produtos de entrada e saída e o mapeamento das fases nas atividades 
mínimas requeridas pela ISO/IEC 15504 e nas fases do SCAMPI, estão relacionados na Figura 3.

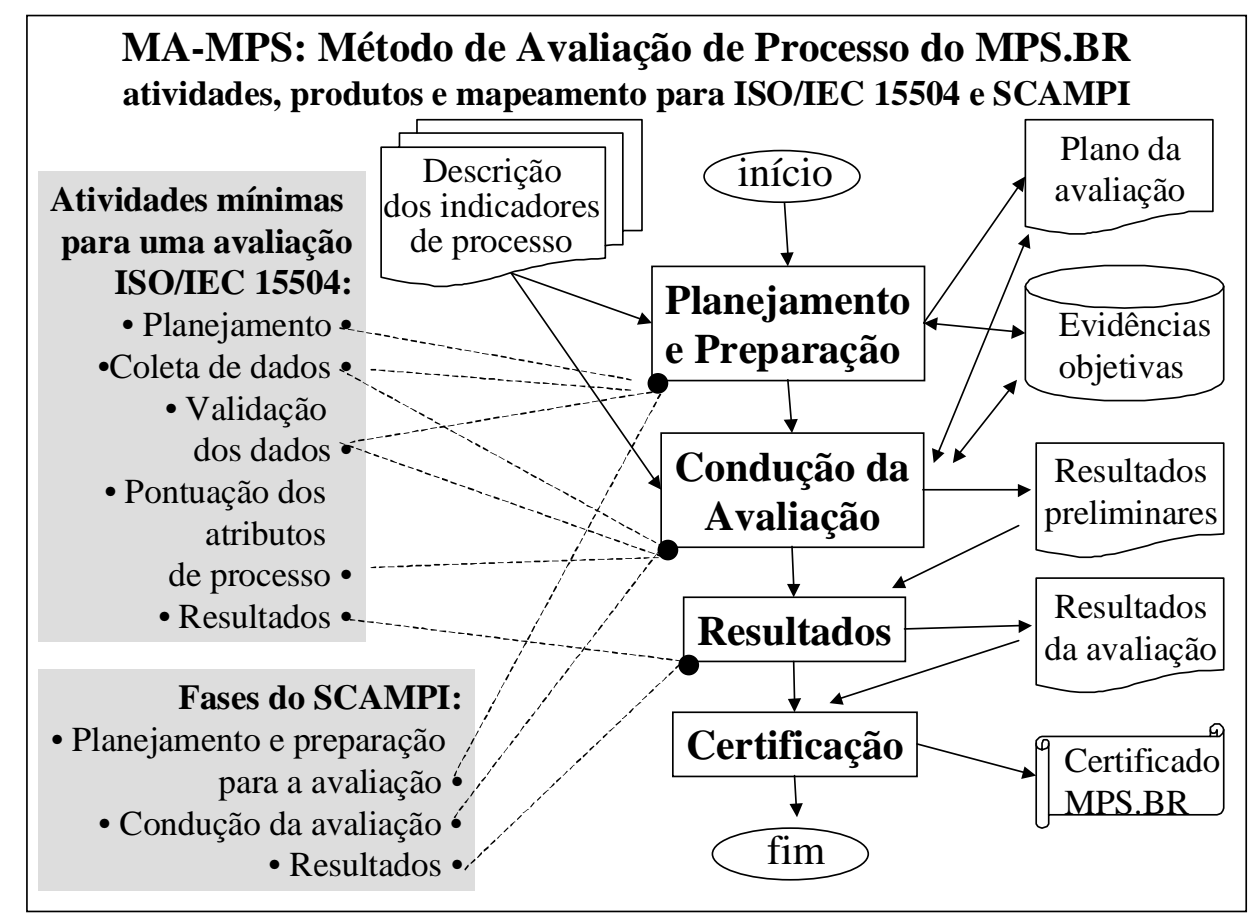

Figura 3. Fluxo geral do processo de avaliação

\subsection{Guia de Aquisição MPS}

\subsubsection{Aquisição de Software}

A aquisição de Software e Serviços Correlatos (S\&SC) é um processo complexo, principalmente no que diz respeito à caracterização dos requisitos necessários ao $\mathrm{S} \& \mathrm{SC}$ e às condições envolvidas na contratação; como, por exemplo, qualidade esperada, forma de aceitação, gestão de mudanças, artefatos esperados, etc. Este ambiente apresenta riscos para as partes envolvidas e, como decorrência, é comum a ocorrência de sérios conflitos na relação entre fornecedores e adquirentes de software. Diante deste cenário, foram empreendidas várias iniciativas internacionais com vistas a tornar este processo mais previsível e com melhores resultados para os envolvidos, resultando, como conseqüência, desde padrões específicos para grandes organizações compradoras de software até normas internacionais que visam orientar relações técnicas e comerciais [Alves 2004].

A elaboração do Guia de Aquisição levou em conta documentos resultantes destes trabalhos, além de considerar relacionamentos do processo de aquisição com aspectos definidos através do MR-MPS. Como a implementação do MR-MPS está perfeitamente relacionada aos processos da norma ISO/IEC 12207 e suas emendas, o Guia de Aquisição, buscando consistência entre os documentos, também está baseado no processo de aquisição daquela norma internacional. O Guia de Aquisição fornece informações complementares à norma ISO/IEC 12207, identificando o relacionamento entre os processos das normas ISO/IEC 12207 e IEEE STD 1062:1998. 


\section{Simpósio Brasileiro de Qualidade de Software}

Um outro aspecto importante a ser considerado refere-se à aplicação de um processo de aquisição envolvendo organizações que estejam adotando o MR-MPS. O documento apresenta algumas facilidades e simplificações que poderão ser aplicadas quando o processo de aquisição envolver organizações fornecedoras de software que estejam adotando o MR-MPS.

O Guia de Aquisição é destinado, mas não está limitado, a organizações interessadas na aquisição de software, inclusive quando estiverem envolvidos fornecedores de software que adotem o MR-MPS para melhoria de seus processos de software. Destina-se também, como uma referência, a organizações desenvolvedoras de software que pretendam estar preparadas para participar de processos de seleção em conformidade com o estabelecido no guia.

\subsubsection{Processo de Aquisição}

O propósito do processo de aquisição, definido no Guia de Aquisição, é obter um S\&SC que satisfaça a necessidade expressa pelo cliente. Este processo inicia com a identificação de uma necessidade do cliente e encerra com a aceitação do produto ou serviço.

Como resultado da implementação bem sucedida do processo de aquisição:

- as necessidades de aquisição, as metas, os critérios de aceitação do S\&SC e as estratégias de aquisição são definidos;

- um contrato que expresse claramente a expectativa, as responsabilidades e as obrigações de ambos (cliente e fornecedor) é desenvolvido;

- um S\&SC que satisfaz a necessidade expressa pelo cliente é adquirido;

- a aquisição é monitorada de forma que as condições especificadas, tais como custo, cronograma e qualidade, são atendidas;

- os produtos entregues pelo fornecedor são aceitos; e

- qualquer pendência identificada tem uma conclusão satisfatória, conforme acordado entre o cliente e o fornecedor.

Conforme mostra a Figura 4, este processo está descrito no Guia de Aquisição através de quatro subprocessos: Preparação da aquisição; Seleção do fornecedor; Monitoração do fornecedor; e Aceitação pelo cliente. 


\begin{tabular}{|c|c|}
\hline $\begin{array}{c}\text { Preparação da } \\
\text { aquisição }\end{array}$ & $\begin{array}{ll}\text { 1. } & \text { Estabelecer necessidade } \\
\text { 2. } & \text { Definir requisitos } \\
\text { 3. } & \text { Revisar requisitos } \\
\text { 4. } & \text { Desenvolver estratégia de aquisição } \\
\text { 5. } & \text { Definir critérios de seleção }\end{array}$ \\
\hline $\begin{array}{l}\text { Seleção do } \\
\text { fornecedor }\end{array}$ & $\begin{array}{l}\text { 1. Avaliar capacidade de fornecedores } \\
\text { 2. Selecionar fornecedor } \\
\text { 3. Preparar e negociar contrato }\end{array}$ \\
\hline $\begin{array}{l}\text { Monitoração do } \\
\text { fornecedor }\end{array}$ & $\begin{array}{ll}\text { 1. } & \text { Estabelecer e manter comunicações } \\
\text { 2. } & \text { Trocar informação sobre progresso técnico } \\
\text { 3. Inspecionar desenvolvimento com fornecedor } \\
\text { 4. Monitorar aquisição } \\
\text { 5. Obter acordo quanto a alterações } \\
\text { 6. Acompanhar problemas }\end{array}$ \\
\hline $\begin{array}{l}\text { Aceitação pelo } \\
\text { cliente }\end{array}$ & $\begin{array}{l}\text { 1. Definir critérios de aceitação } \\
\text { 2. Avaliar produto entregue } \\
\text { 3. Manter conformidade com o contrato } \\
\text { 4. Aceitar o software }\end{array}$ \\
\hline
\end{tabular}

\section{Figura 4. Subprocessos e atividades do processo de aquisição} quadros:

Cada um dos subprocessos está detalhado no Guia de Aquisição pelos seguintes

- Atividades previstas: identifica e descreve as atividades necessárias para atingir os objetivos e obter os resultados previstos para o subprocesso;

- Produtos requeridos: relaciona os insumos que serão necessários para executar cada atividade prevista no subprocesso;

- Produtos gerados: relaciona os produtos de cada atividade prevista no subprocesso. Para alguns destes produtos há referências de modelos descritos em anexos deste Guia; e

- Contratações com organizações avaliadas segundo o MR-MPS: identifica características que poderão facilitar a execução de algumas atividades do subprocesso, desde que o fornecedor tenha sido avaliado segundo o MR-MPS ou outro modelo equivalente.

Além da definição e detalhamento do processo de aquisição de S\&SC, o Guia de Aquisição trata de outros aspectos relevantes, como os problemas comuns que ocorrem na aquisição, aquisição de software livre/código aberto, aquisição e a engenharia de software baseada em componentes, e a evolução do nível de capacidade no processo de aquisição de S\&SC. 


\section{Primeiros Resultados Alcançados no Projeto MPS.BR (Dez2003- Mar2005)}

\subsection{Meta Física 1: Desenvolvimento e Aprimoramento do Modelo MPS}

- Dez2003-Jul2004: Desenvolvimento de uma versão preliminar do Modelo MPS, compatível com o CMMI.

- Ago2004-Mar2005: Desenvolvimento da versão 1.0 do Modelo MPS, compatível com o CMMI e, adicionalmente, em conformidade com as normas ISO/IEC 12207 e ISO/IEC 15504. Produção do Guia Geral (a ser publicada em Abr2005), Guia de Avaliação (a ser validada até Jul2005) e Guia de Aquisição (a ser publicada em Mai2005).

- Mai-Dez2004: Realização de 12 Cursos de Introdução ao Modelo MPS, em 11 cidades, com 637 participantes. Realização de 3 Provas de Conhecimento para Consultores de Implementação do Modelo MPS, simultaneamente em 12 cidades, com 137 aprovados.

- Nov2004-Mar2005: Autorizadas pela SOFTEX, dependendo de assinatura de convênio, as quatro primeiras Instituições Implementadoras do Modelo MPS (IIMPS) - COPPE/UFRJ do Rio de Janeiro/RJ, ITS de São Paulo/SP, UFLA de Lavras/MG e UNIFOR de Fortaleza/CE.

\subsubsection{Meta Física 2: Implementação e Avaliação do Modelo MPS em Empresas}

- Dez2003-Mar2005: Realização de experiências-piloto de Implementação do Modelo MPS em grupos de empresas - 17 empresas no Rio de Janeiro/RJ (RIOSOFT junto com COPPE/UFRJ), 28 empresas no Recife/PE (CESAR é Agente SOFTEX) e 5 empresas em Campinas/SP (Agente SOFTEX junto com CenPRA).

- Set2004-Mar2005: Primeiras ações internacionais, com apresentações do Projeto MPS.BR e Modelos do MPS na Argentina, Peru e Portugal, e busca de apoio do BID.

\section{Conclusão}

Neste artigo apresentamos a versão 1.0 do Modelo de Referência e do Método de Avaliação para Melhoria de Processo de Software (MR-MPS e MA-MPS) e uma descrição dos seus três guias, que estão sendo desenvolvidos no Projeto MPS.BR melhoria de processo do software Brasileiro.

Este projeto visa a melhoria de processo de software em todas as regiões do Brasil, com foco nas pequenas e médias empresas, a um custo acessível . É um empreendimento magno no setor de software Brasileiro, com forte interação Universidade-Empresas-Governo, implicando em mudança cultural significativa.

Sobretudo, tem se mostrado acertada a opção pela criação no Brasil de modelos próprios, um Modelo de Referência de Processo (MR-MPS) e um Modelo de Avaliação de Processo (MA-MPS), que atendam à realidade nacional, estejam em conformidade 


\section{Simpósio Brasileiro de Qualidade de Software}

com as normas internacionais ISO/IEC 12207 e ISO/IEC 15504, e sejam compatíveis com outros modelos renomados, tais como o CMMI.

O Modelo MPS tem um grande potencial de replicabilidade em muitas cidades no Brasil e em outros países com características similares no que se refere ao setor de software, como na América Latina [Weber et al 2004a e 2004b].

Como principais resultados, até agora, destacamos:

- um amplo debate sobre o Projeto MPS.BR e o Modelo MPS, com extraordinária receptividade em todas as regiões do país e em organizações de diferentes portes, privadas e governamentais;

- o envolvimento de uma grande equipe, com representantes de várias regiões brasileiras;

- o desenvolvimento e aprimoramento do Modelo MPS, documentado nos três Guias MPS, com grande agregação de valor e impacto tecnológico;

- a capacitação de centenas de pessoas, por meio de Cursos e Provas MPS em 2004, com diversas lições aprendidas;

- a autorização das quatro primeiras Instituições Implementadoras do Modelo MPS (II-MPS);

- a realização de experiências-piloto de Implementação do Modelo MPS em grupos de empresas no Rio de Janeiro, Recife e Campinas, também com muitas lições aprendidas.

Os principais desafios, em 2005, são:

- comprometer com o Projeto MPS.BR e o Modelo MPS mais pessoas qualificadas, instituições experientes, empresas e organizações interessadas;

- publicar e continuar aprimorando os três Guias MPS;

- realizar o calendário 2005 de Cursos e Provas MPS, levando em conta as lições aprendidas em 2004;

- conceder autorização para novas Instituições Implementadoras do Modelo MPS (II-MPS) e, no final de 2005, para Instituições Avaliadoras do Modelo MPS (IA-MPS);

- realizar experiências-piloto de Avaliação do Modelo MPS previstas no Rio de Janeiro, Recife e Campinas, de Mai-Jul2005, e outras avaliações em locais diversos, de Ago/Dez2005;

- criar novos grupos de empresas para Implementação do Modelo MPS no Rio de Janeiro, Recife, Campinas e em outros locais, tais como Belo Horizonte, Brasília, Fortaleza, Porto Alegre e São Paulo, considerando as lições aprendidas em 2004;

- em paralelo, divulgar o Guia de Aquisição MPS e personalizá-lo para domínios e organizações, públicos e privados, interessados na melhoria do processo de aquisição de Software e Serviços Correlatos (S\&SC). 


\section{Simpósio Brasileiro de Qualidade de Software}

\section{Agradecimentos}

Colaboraram na produção da versão 1.0 do Guia Geral: Cristina Machado e Ana Regina Rocha (editoras), Adriano Albuquerque, Ana Carolina Natali, Clênio F. Salviano, Danilo Scalet, Edson Almeida, Gleison Souza, Marcelo Pessoa, Mariano Montoni, Odisnei Galarraga, Paula Mian, Sávio Figueiredo, Sheila Reinehr, Tayana Conte e Teresa Maciel. O desenvolvimento da versão 1.0 do Guia de Avaliação conta com os seguintes colaboradores: Cristina Machado e Ana Regina Rocha (editoras), Alessandra Anacleto, Clênio Salviano, Danilo Scalet, Luís Carlos de Almeida Oliveira, Márcio Pecegueiro do Amaral e Sarah Kohan. Participaram da elaboração da versão 1.0 do Guia de Aquisição: Danilo Scalet (editor), Ana Guerra, Ângela Alves, Clênio F. Salviano, Lúcia Nigro e Regina Colombo.

Os três Guias MPS são revisados pelos membros da ETM e serão aprimorados periodicamente, com suas diferentes versões mantidas sob controle.

MR-MPS, MA-MPS, MN-MPS e MPS.BR são marcas da SOFTEX.

$\mathrm{CMM}^{\mathrm{SM}}, \mathrm{CMMI}^{\mathrm{SM}}$ e SCAMPI ${ }^{\mathrm{SM}}$ são marcas do SEI/CMU.

\section{Referências Bibliográficas}

Alves, A. M., Guerra, A. Aquisição de Produtos e Serviços de Software. Rio de Janeiro: Elsevier, 2004. 213p.

Anacleto, A., von Wangenheim, C. G., Salviano, C. F., Savi, R. "A Method for Process Assessment in Small Software Companies", In: Proceedings of SPICE 2004: The Fourth International SPICE Conference. Lisbon, Portugal: April 28-29, 2004. pp. 69-76.

Chrissis, M. B., Konrad, M., Shrum, S. CMMI ${ }^{\mathrm{SM}}$ : Guidelines for Process Integration and Product Improvement. Addison-Wesley, 2003.

IEEE STD 1062:1998. IEEE Software Engineering Standards Collection. IEEE Recommended Practice for Software Acquisition, IEEE STD 1062 Edition. New York, NY: 1998. 43p.

ISO/IEC 12207:1995/Amd 1:1998/Amd 2:2002. Technology Information - Software Life Cycle Processes.

ISO 9001:2000. Quality Management Systems Requirements.

ISO/IEC 15504. Technology Information - Process Assessment. Part 1 - Concepts and vocabulary; part 2 - Performing an assessment; part 3 - Guidance on performing an assessment; part 4 - Guidance on use for process improvement and process capability determination; and part 5 - An exemplar process assessment model.

MCT/SEPIN - Ministério da Ciência e Tecnologia, Secretaria de Política de Informática. Qualidade e Produtividade no Setor de Software Brasileiro 2001. Brasília: 2001.

Kohan, S. QuickLocus: Proposta de um método de avaliação de processo de desenvolvimento de software em pequenas organizações. Dissertação de mestrado, Instituto de Pesquisas Tecnológicas do Estado de São Paulo - IPT, 2003. 234 p. 


\section{Simpósio Brasileiro de Qualidade de Software}

SEI - Software Engineering Institute, Carnegie Mellon University. Standard CMMI ${ }^{\mathrm{SM}}$ Appraisal Method for Process Improvement $\left(\mathrm{SCAMPI}^{\mathrm{SM}}\right)$, Version 1.1: Method Definition Document, CMU/SEI-2001-HB-001, 2001.

van Loon, H. Process Assessment and ISO/IEC 15504: a reference book. The Kluwer International Series in Engineering and Computer Science, Volume 775. Springer, 2004a. p. 85.

van Loon, H. Process Assessment and Improvement: a pratical guide for managers, quality professionals and assessors. The Kluwer International Series in Engineering and Computer Science, Volume 776. Springer, 2004b. pp. 10-11.

Veloso, F., Botelho, A. J., Tschang, A., Amsden, A. Slicing the Knowledge-based Economy in Brazil, China and India: a tale of 3 software industries. Report, MIT Massachusetts Institute of Technology, Mass, September 2003.

Weber, K. C., Rocha, A. R., Alves, A., Ayala, A. M., Gonçalves, A., Paret, P., Salviano, C., Machado, C. F., Scalet, D., Petit, D., Araújo, E., Barroso, M. G., Oliveira, K., Oliveira, L. C. A., Amaral, M. P., Campelo, R. E. C., Maciel, T. "Modelo de Referência para Melhoria de Processo de Software: uma abordagem brasileira", In: Proceedings of the XXX Conferencia Latinoamericana de Informatica ( CLEI 2004). Arequipa, Peru: septiembre 2004a.

Weber, K. C., Rocha, A. R., Rouiller, A. C., Crespo, A., Alves, A., Ayala, A., Gonçalves, A., Paret, B., Vargas, C., Salviano, C., Machado, C., Scalet, D., Petit, D., Araújo, E., Maldonado, J. C., Oliveira, K., Oliveira, L. C., Girão, M., Amaral, M., Campelo, R., Maciel, T. "Uma Estratégia para Melhoria de Processo de Software nas Empresas Brasileiras", In: Proceedings of the Fifth Conference for Quality in Information and Communications Technology (QUATIC'2004). Porto, Portugal: October 2004b. pp. 73-78. 